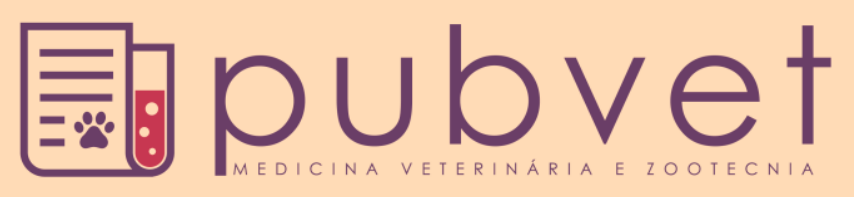

https://doi.org/10.22256/pubvet.v12n4a77.1-12

\title{
Efeitos de três protocolos farmacológicos no controle da dor em bezerras holandesas descornadas com ferro quente
}

\author{
Jackson Barros do Amaral ${ }^{\ominus *}$, Luciandra Macedo de Toledo ${ }^{\ominus 1}$, Luís Alberto \\ Ambrósio ${ }^{\ominus}$, Flávia Augusta de Oliveira ${ }^{\ominus}$, Guilherme Trevisan ${ }^{\ominus}$ \\ ${ }^{1}$ Centro de Análise e Pesquisa Tecnológica dos Agronegócios de Bovinos de Leite, Instituto de Zootecnia, Secretaria de Agricultura e \\ Abastecimento, Nova Odessa/São Paulo, Brasil. \\ ${ }^{2}$ Universidade Federal de Goiás - UFG, Jataí - Goiás, Brasil. \\ ${ }^{3}$ Médico Veterinário, Mestrando da Faculdade de Medicina Veterinária e Zootecnia “Julio de Mesquita Filho”- Distrito de Rubião Jr., s/n- \\ Botucatu, São Paulo, Brasil. \\ *Autor Correspondente - Centro de Análise e Pesquisa Tecnológica dos Agronegócios de Bovinos de Leite, Instituto de Zootecnia - IZ, Rua \\ Heitor Penteado, 56, CP. 60, CEP 13.460-000, Nova Odessa/SP, Brasil. Tel: +5519 3466-9435. \\ E-mail: jackson@iz.sp.gov.br
}

\begin{abstract}
RESUMO. Este trabalho teve como objetivo estudar a eficiência de três protocolos no controle da dor pela avaliação do comportamento na descorna de bezerras. Utilizou-se 30 bezerras distribuídas em três grupos para aplicação dos protocolos I, II e III, em delineamento inteiramente ao acaso. O protocolo I (controle) utilizou $7 \mathrm{mg} / \mathrm{kg}$ de cloridrato de lidocaína para bloqueio cornual. No protocolo II os animais foram submetidos ao mesmo procedimento do protocolo I acrescido da aplicação IM de $0,04 \mathrm{mg} / \mathrm{kg}$ de xilazina. No protocolo III os animais foram submetidos aos mesmos procedimentos dos protocolos I e II acrescidos da aplicação IM de $1,1 \mathrm{mg} / \mathrm{kg}$ de flunixina-meglumina. Avaliou-se o comportamento no momento da descorna e após. A diferença entre a vocalização durante o procedimento da descorna foi significativa $(\mathrm{P}=0,0229)$ entre os protocolos, sendo a frequência de PII de $70 \%$ e do PI de $10 \%$ dos animais. No período pós-descorna evidenciou-se diferença significativa no comportamento dos animais dos protocolos II e III, os quais deitaram menos vezes quando comparados com o protocolo I. Conclui-se que os protocolos II e III foram mais eficientes no controle da dor, indicando que o uso do sedativo isoladamente ou associado ao anti-inflamatório contribuiu no bem-estar animal.
\end{abstract}

Palavras chave: excisão de corno, anestesia local, bem-estar animal, bovinos, etologia clínica

\section{Effects of three pharmacological protocols in pain control in holstein calves by hot-iron dehorning}

\begin{abstract}
The objective of this study was to study the efficiency of three protocols in pain control by evaluating behaviour in calf dehorning. Thirty heifers were distributed in three groups for the application of protocols I, II and III, in a completely randomized design. Protocol I (control) utilized $7 \mathrm{mg} / \mathrm{kg}$ of lidocaine hydrochloride was applied on cornual nerve block. In protocol II the animals were submitted to the same procedure of protocol I plus IM application of $0.04 \mathrm{mg} / \mathrm{kg}$ of xylazine. In protocol III the animals were submitted to the same procedures of protocols I and II plus the IM application of $1.1 \mathrm{mg} / \mathrm{kg}$ of flunixin-meglumine. The behaviour was evaluated before and after dehorning. The difference between the vocalization during the dehorning procedure was significant $(\mathrm{P}=0.0229)$ between the protocols, with the frequency of PII being $70 \%$ and of the IP of $10 \%$ of the animals. In the post-dehorning period, there was a significant difference in animal behaviour of protocols II and III, which lay down less compared to protocol I. It
\end{abstract}


was concluded that protocols II and III were more efficient in pain control, indicating that the use of sedative alone or associated with anti-inflammatory has contributed to improve animal welfare.

Keywords: animal welfare, bovine, clinical ethology, horn excision, local anesthesia

\section{Efectos de tres protocolos farmacológicos en el control del dolor en terneras holandesas descornadas con hierro caliente}

RESUMEN. El objetivo de este trabajo fue estudiar la eficacia de tres protocolos en el control del dolor mediante la evaluación del comportamiento en el descuerne de terneras. Treinta novillas se distribuyeron en tres grupos para la aplicación de los protocolos I, II y III, en un diseño completamente aleatorizado. Protocolo I (control) Se aplicaron $7 \mathrm{mg} / \mathrm{kg}$ de clorhidrato de lidocaína en el bloqueo del nervio cornual. En el protocolo II, los animales se sometieron al mismo procedimiento del protocolo I más la aplicación IM de 0,04 mg / $\mathrm{kg}$ de xilazina. En el protocolo III, los animales se sometieron a los mismos procedimientos de los protocolos I y II más la aplicación IM de $1,1 \mathrm{mg} / \mathrm{kg}$ de flunixina-meglumina. El comportamiento fue evaluado durante y después de descornado. La diferencia entre la vocalización durante el procedimiento de descornado fue significativa $(\mathrm{P}=0.0229)$ entre los protocolos, con una frecuencia de PII del $70 \%$ y de PI del $10 \%$ de los animales. En el período posterior al descornado, hubo una diferencia significativa en el comportamiento animal de los protocolos II y III, que establecen menos en comparación con el protocolo I. Se concluyó que los protocolos II y III fueron más eficientes en el control del dolor, lo que indica que el uso del sedante solo o asociado con antiinflamatorios contribuyó a mejorar el bienestar de los animales.

Palabras clave: bienestar animal, bovino, etología clínica, escisión de cuerno, anestesia local

\section{Introdução}

Os animais de produção estão sujeitos a diversas atividades de manejo que afetam direta ou indiretamente a integridade física devido a procedimentos clínico-cirúrgicos que causam dor aguda ou superagudo. Neste sentido, a possibilidade da ocorrência de dor nos animais está presente em todos os sistemas de criação da pecuária, sendo este um tema que vem ganhando destaque na comunidade científica nos últimos anos (Luna, 2006).

Apesar da descorna com ferro quente ser segura é considerada um procedimento muito doloroso, tendo em vista que o calor excessivo aplicado causa lesões por queimadura nos tecidos, incluindo o ósseo. Os benefícios do uso de anestésicos e analgésicos a descorna de bezerros vem sendo realizada rotineiramente sem aplicação prévia destes fármacos em vários países (Vickers et al., 2005, Stewart et al., 2009).

No trabalho realizado por Misch et al. (2007) foi demonstrado que de $78 \%$ dos produtores de leite apenas $22 \%$ utilizavam anestésicos locais e que $92 \%$ dos veterinários utilizavam este fármaco para realizar descorna.

No Brasil, Lorena (2010) demonstrou por questionários aplicados aos produtores rurais que o controle da dor em animais de produção ainda continua sendo um desafio, onde o alto custo dos analgésicos é considerado um problema. De forma semelhante um estudo canadense aplicado por meio de questionários a produtores rurais evidenciou que $90 \%$ dos procedimentos de castração e $85 \%$ das descornas de bezerros não utilizam estratégias contra a (Moggy et al., 2017).

Quando a descorna é realizada com anestesia local associada à sedação e anti-inflamatório bloqueia-se a dor durante e após este procedimento, facilita a contenção dos animais em posição quadrupedal e contribui para melhorar o bem-estar dos animais. Fundamentado nesta hipótese este trabalho teve como objetivo estudar a eficiência de três protocolos farmacológicos no controle da dor pela avaliação do comportamento de bezerras da raça Holandesa descornados com ferro quente. 


\section{Material e métodos}

Este trabalho foi previamente aprovado pela Comissão de Ética no Uso de Animais do Instituto de Zootecnia (CEUA/IZ), registrado no Protocolo $\mathrm{n}^{\circ} 131$. O experimento foi realizado no Centro de Análises e Pesquisas Tecnológicas dos Agronegócios de Bovinos de Leite, CAPTA Bovinos de Leite, do Instituto de Zootecnia da Secretaria de Agricultura e Abastecimento do Estado de São Paulo - SAA/SP, Brasil.

Foram utilizadas 30 bezerras da raça Holandesa, divididos em três grupos de 10 animais submetidos ao controle de doenças infectocontagiosas e parasitárias e em boas condições clínicas para aplicação dos protocolos PI, PII e PIII, em delineamento inteiramente ao acaso. No período pré-operatório os animais foram submetidos a jejum de 12 horas, pesagens para dosagens de fármacos e tricotomias das regiões cornuais para facilitar os procedimentos de descorna e cicatrizações das feridas cirúrgicas. O período transoperatório foi conduzido após antissepsia das regiões cornuais com álcool iodado e pelos procedimentos de descorna, seguindo os protocolos PI, PII e PIII. No período pósoperatório os animais foram submetidos a todos os procedimentos de tratamento preventivo e curativo das feridas cirúrgicas.

No Protocolo I (PI) foi descrito pelo controle no qual os animais receberam anestesia local por meio do bloqueio perineural do nervo cornual pela aplicação de $7 \mathrm{mg} / \mathrm{kg}$ de cloridrato de lidocaína a $2 \%$ (Lidovet $^{\circledR}$, Bravet) e $7 \mathrm{mg} / \mathrm{kg}$ do mesmo anestésico infiltrado em pontos circundando cada corno, abaixo da linha de transição do tecido queratocórneo. No Protocolo II (PII) os animais foram submetidos ao mesmo Protocolo I, associado a $0,04 \mathrm{mg} / \mathrm{kg}$ de cloridrato de xilazina a $2 \%$ (Rompun $^{\circledR}$, Bayer) pela via intramuscular (IM). No Protocolo III (PIII) os animais foram submetidos ao mesmo Protocolo II associado a 1,1 $\mathrm{mg} / \mathrm{kg}$ de flunixina-meglumina (Desflan ${ }^{\circledR}$, Ourofino) pela via IM. Procedeu-se a descorna com os animais em estação, com o pescoço contido na trava de contenção do tronco, sendo a cabeça imobilizada por um funcionário auxiliar. Este método de contenção da cabeça foi necessário para realização da termocauterização com segurança, evitando queimaduras acidentais nos animais e funcionários.

O procedimento de descorna foi realizado nas bezerras com idades entre quatro a cinco meses, portanto, apresentavam pequeno processo cornual o qual foi amputado com uma serra fixada em arco. Imediatamente após a remoção de cada corno realizou-se a termocauterização com ferro quente apropriado para controlar a hemorragia, cauterizar os tecidos da base e remover as aparas. Para evitar complicações como infecções, sinusites catarrais e miíases infiltrativas no período pós-descorna procedeu-se a antibioticoterapia pela da aplicação de $11 \mathrm{mg} / \mathrm{kg}$ de oxitetraciclina (Terramicina ${ }^{\circledR}$ LA, Pfizer) por via IM sendo a mesma dose repetida após 72 horas. Os curativos das feridas operatórias foram realizados diariamente até completar a cicatrização utilizando pomada de ação parasiticida, cicatrizante e repelente (Unguento Pearson ${ }^{\circledR}$ ).

A avaliação comportamental foi realizada durante e após o procedimento de descorna, acordo com a metodologia de Martin et al. (1993). Durante a realização da descorna a avaliação do comportamento foi fundamentada na etologia clínica registrada por meio de vídeos utilizando-se câmera digital marca Sony DSC-W55 acoplada a um tripé para facilitar as filmagens, estabilizar as imagens e captar o som da vocalização. As imagens foram assistidas posteriormente por três observadores treinados para transcrição dos dados para análise.

No momento da descorna a observação foi contínua no qual se registrou movimentos defensivos da cabeça, membros e abanar da cauda. Os movimentos da cabeça foram classificados em reativos e não reativos durante a contenção da mesma. Consideram-se movimentos defensivos dos membros todas e quaisquer reatividades dos mesmos, assim como, movimentos de abanar da cauda presente ou ausente. $\mathrm{Na}$ vocalização avaliaram-se todos os sons audíveis gerados na laringe, propagados pelas cavidades ressonantes, incluindo boca e narinas, sendo classificados em breve (duração de até dois segundos) e prolongado (duração acima de dois segundos). Após o procedimento de descorna os animais permaneceram em um piquete medindo $20 \times 40$ metros, onde foram realizadas observações comportamentais por dois dias consecutivos com amostragem focal e instantânea a cada cinco minutos, realizada por observadores treinados. As observações foram realizadas duas vezes ao dia, das $9 \mathrm{~h}$ às $11 \mathrm{~h}$ e das $14 \mathrm{~h}$ às $16 \mathrm{~h}$, totalizando quatro turnos de observações. Observaram-se os comportamentos de postura (deitado ou em pé) e as atividades dos animais (coçando, pastando, sem atividade aparente, ingerindo água). 


\section{Análise estatística}

A análise estatística para avaliação da associação entre as proporções de animais que manifestaram os comportamentos dos respectivos protocolos farmacológicos durante o procedimento da descorna foi realizada pela aplicação do Teste Exato de Fisher-FreemanHalton para tabela de contingência $2 \times 3$, o qual é uma extensão do Teste Exato de Fisher para tabela de contingência $2 \times 2$. Neste caso a hipótese nula $\left(\mathrm{H}_{0}\right)$ de independência entre a manifestação do comportamento (Sim/Não) e o protocolo farmacológico (PI, PII e PIII) foram rejeitados quando $\mathrm{P}<0,05$. A análise do comportamento do período pós-descorna nos três protocolos foi realizada pelo teste de Kruskal-Wallis $(\mathrm{H})$ e pelo teste Q de Cochran, utilizando-se os softwares Statistical Analysis System 9.1 (SAS, 2004)) Proc FREQ e Bioestat 5.0. Nas Análises de Variâncias de Kruskal-Wallis (H) e no teste Q de Cochran foi considerado o nível de confiança de 95\%. $(\mathrm{P}<0,05)$.

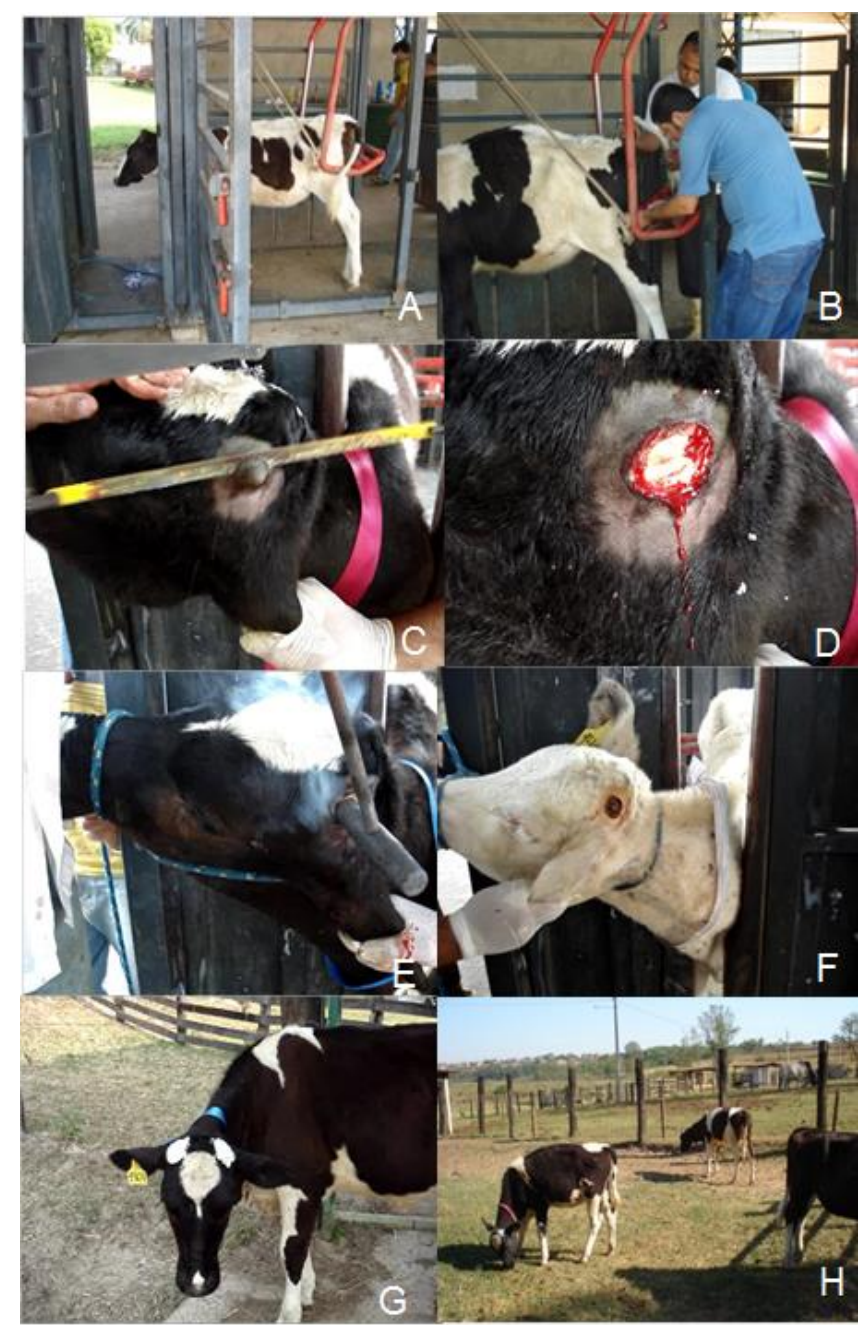

Figura 1. Fotografias dos bezerros durante e após os procedimentos de descorna: A- Contenção; B- Aplicação intramuscular de fármacos; C- Descorna; D- Hemorragia pós-descorna; E Termocauterização; F- Descorna concluída; G- Curativos da ferida cirúrgica; H- Avaliação do comportamento pós-descorna.

\section{Resultados}

Houve diferença significativa para frequência da vocalização durante descorna em relação aos tratamentos PI e PII, entretanto, os demais comportamentos não apresentaram diferenças significativas (Tabela 1). A vocalização com emissão de mugidos ocorreu em $70 \%$ dos animais do protocolo II e $40 \%$ dos animais do protocolo III, sendo ambos maiores que no protocolo controle (10\%), Tabela 1.

Ocorreram diferenças significativas entre os procedimentos nos turnos, sendo: ingestão de água no geral $(\mathrm{Q}=-11,9 ; \mathrm{P}=0,008)$, ingestão de alimentos no protocolo II $(\mathrm{Q}=-9,265 ; \mathrm{P}=0,026)$, autolimpeza corporal no protocolo II $(\mathrm{Q}=$ - 
$8,4146 ; \mathrm{P}=0,0382)$ e ruminando nos protocolos I e III $(\mathrm{Q}=-12,4748 ; \mathrm{P}=0,0059)$, (Tabelas 2 - 4).
Para os demais comportamentos não houve diferenças significativas nos turnos.

Tabela 1. Comportamento dos animais durante o procedimento de descorna em função dos protocolos farmacológicos, em frequência de bezerras que apresentaram o comportamento (Sim).

\begin{tabular}{|c|c|c|c|c|c|c|c|c|c|}
\hline \multirow[t]{2}{*}{ Protocolos ${ }^{1}$} & \multirow{2}{*}{$\begin{array}{c}\text { Animais } \\
\mathrm{n}\end{array}$} & \multicolumn{2}{|c|}{ Vocalização\# } & \multicolumn{2}{|c|}{$\begin{array}{l}\text { Alteração } \\
\text { da Cabeça }\end{array}$} & \multicolumn{2}{|c|}{ Temperamento } & \multicolumn{2}{|c|}{$\begin{array}{l}\text { Alteração } \\
\text { da Cauda }\end{array}$} \\
\hline & & Sim & $\%$ & Sim & $\%$ & Sim & $\%$ & Sim & $\%$ \\
\hline (PI) & 10 & $1^{\mathrm{A}}$ & 10,0 & 9 & 90,0 & 5 & 50,0 & 7 & 70,0 \\
\hline (PII) & 10 & $7^{\mathrm{B}}$ & 70,0 & 7 & 70,0 & 2 & 20,0 & 2 & 20,0 \\
\hline (PIII) & 10 & $4^{\mathrm{AB}}$ & 40,0 & 7 & 70,0 & 3 & 30,0 & 4 & 40,0 \\
\hline
\end{tabular}

* = Diferença significativa a 5\% (P<0,05), Teste Exato de Fisher-Freeman-Halton; = Letras diferentes indicam diferenças significativas a 5\% (P < 0,05), Teste Exato de Fisher-Freeman Halton - desdobramento de contraste (2x3). 1. PI Anestesia; PII Anestesia Sedação; PIII Anestesia Sedação Anti-inflamatório

\section{Discussão}

Alterações comportamentais com altas frequências foram observadas na aplicação dos protocolos de descorna, variando de $70 \%$ a $90 \%$ na cabeça, de 20 a $50 \%$ no temperamento e 20 a $70 \%$ na cauda, que demonstra afetar o bem-estar animal. No protocolo I (controle) teve menor proporção de animais que vocalizaram em relação aos protocolos II e III. Desta forma, não houve associação entre as proporções de animais que manifestaram os comportamentos e os tipos de protocolos, ou seja, os protocolos não diferenciaram na influência dos comportamentos observados. A vocalização ocorrida em $70 \%$ dos animais do protocolo II e $40 \%$ dos animais do protocolo III pode estar associada aos efeitos colaterais do cloridrato de xilazina e não devido à dor, devendo ser investigada em estudos específicos. Apesar destes resultados deve-se levar em consideração que o cloridrato de xilazina é o fármaco mais usado para promover a sedação em grandes animais, especialmente em ruminantes, que são dez vezes mais sensíveis quando comparado com os equinos (Abrahamsen, 2008). Além disso, deve-se considerar também que a associação deste fármaco com os anestésicos locais permite intervenções cirúrgicas mais profundas, obtendo-se, assim, excelentes resultados em procedimentos dolorosos utilizados na prática de campo, como é o caso da descorna. No entanto, devem-se levar em consideração que de acordo com Luna (2006) a avaliação da dor em animais é difícil, sendo o comportamento o componente principal para seu reconhecimento. Segundo este autor os bovinos com dor normalmente vocalizam com grunhidos e urros, rangem os dentes e relutam em se mover, alteram a expressão facial e reduzem a produtividade.
No trabalho realizado por Caray et al. (2015) foi mostrado que bezerros descornados sem xilazina apresentaram estresse, provavelmente devido a dor aguda induzida pela termocauterização. Os autores mostraram ainda que na descorna de bezerros sedados com xilazina vocalizaram tanto quanto aqueles que receberam apenas anti-inflamatório, sugerindo certa intensidade de dor. Quando a sedação com xilazina foi associada à aplicação do antiinflamatório não demonstraram comportamentos que evidenciassem dor. Desta forma, concluíram que a descorna é um procedimento doloroso e que a sedação com xilazina associada ao antiinflamatório pode contribuir para melhorar o bemestar na descorna dos bezerros.

No presente trabalho utilizou-se serra fixada em arco para remoção dos botões cornuais, os quais já estavam iniciando a fusão com os ossos do crânio, devido os animais apresentarem idades entre quatro a cinco meses. De acordo com Massone (1999) o procedimento de descorna com auxílio de serra ou de um fio-serra deve ser realizado sob a aplicação prévia de medicação préanestésica, como a xilazina, tendo em vista que este procedimento causa ruído exagerado e repercussão encefálica, a qual faz com que o animal se debata em decorrência de desconfortos e não devido à dor. Segundo a AVMA (2014) o nervo cornual representa um ramo do nervo trigêmeo e proporciona a sensibilidade da região do corno. Sendo assim, a injeção de anestésico local em torno deste nervo dessensibiliza esta área, sendo um procedimento anestésico importante no controle da dor na descorna de bezerros realizada com ou sem ferro quente. No entanto, no presente trabalho não houve diferença significativa no comportamento de alteração da cabeça entre os 


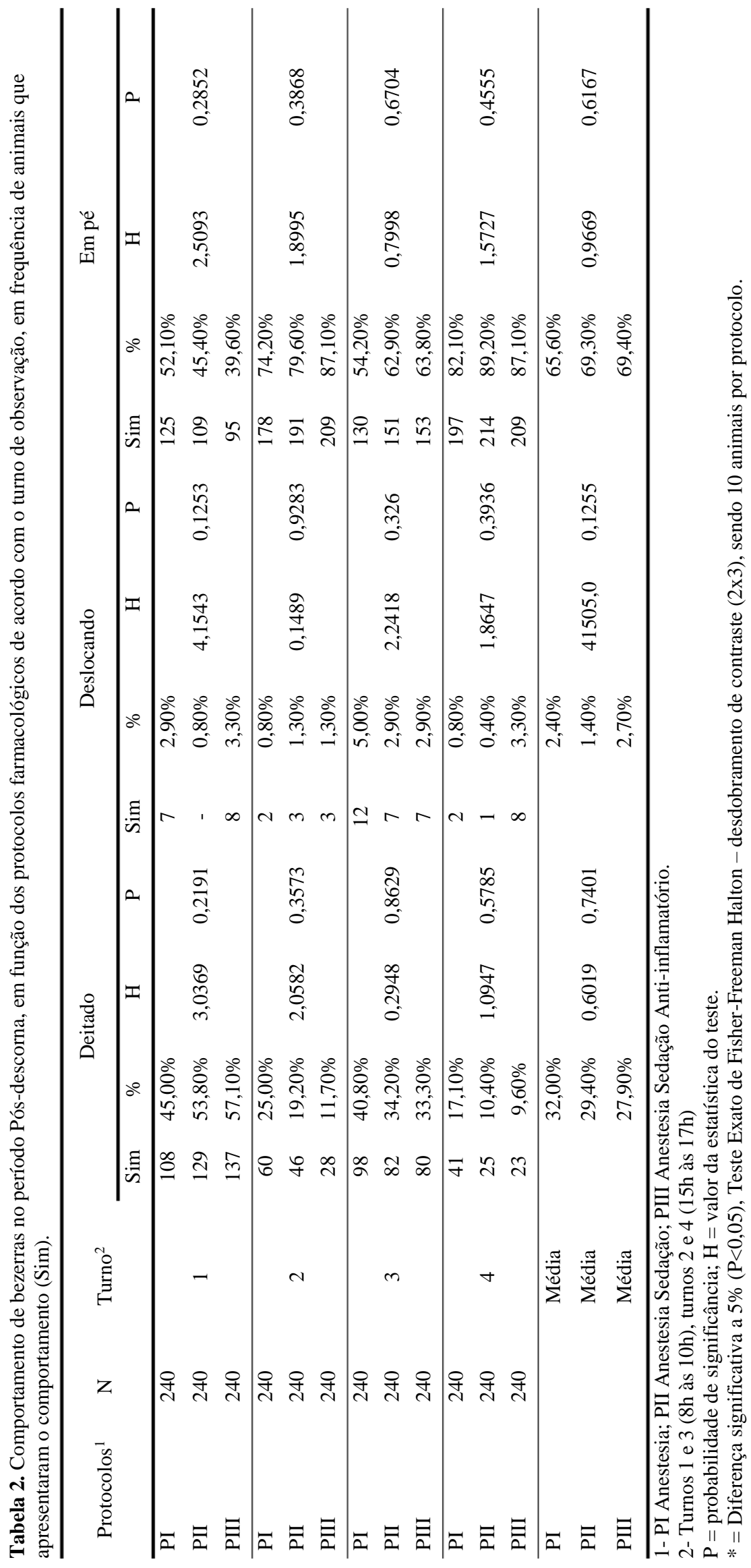




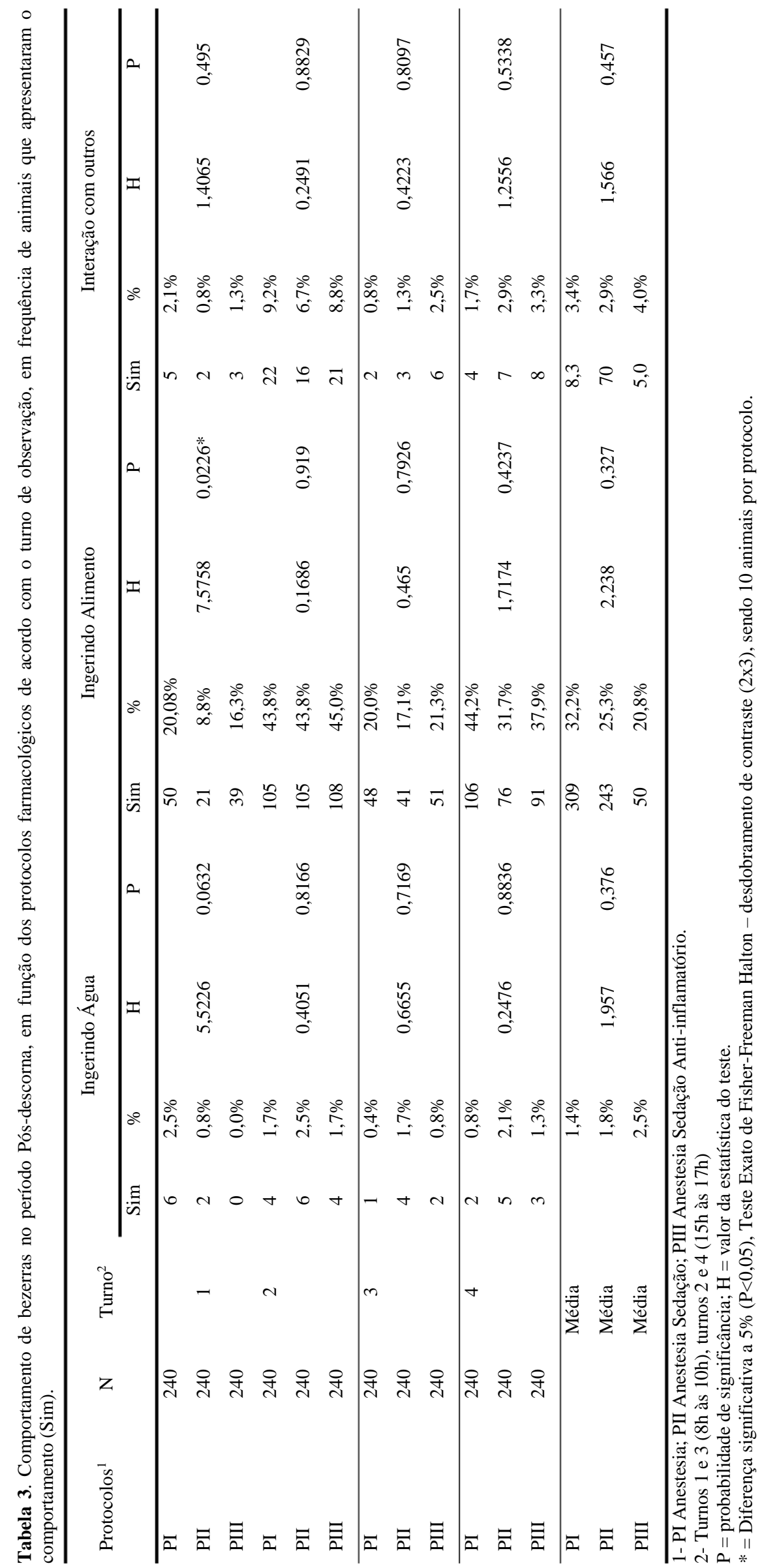




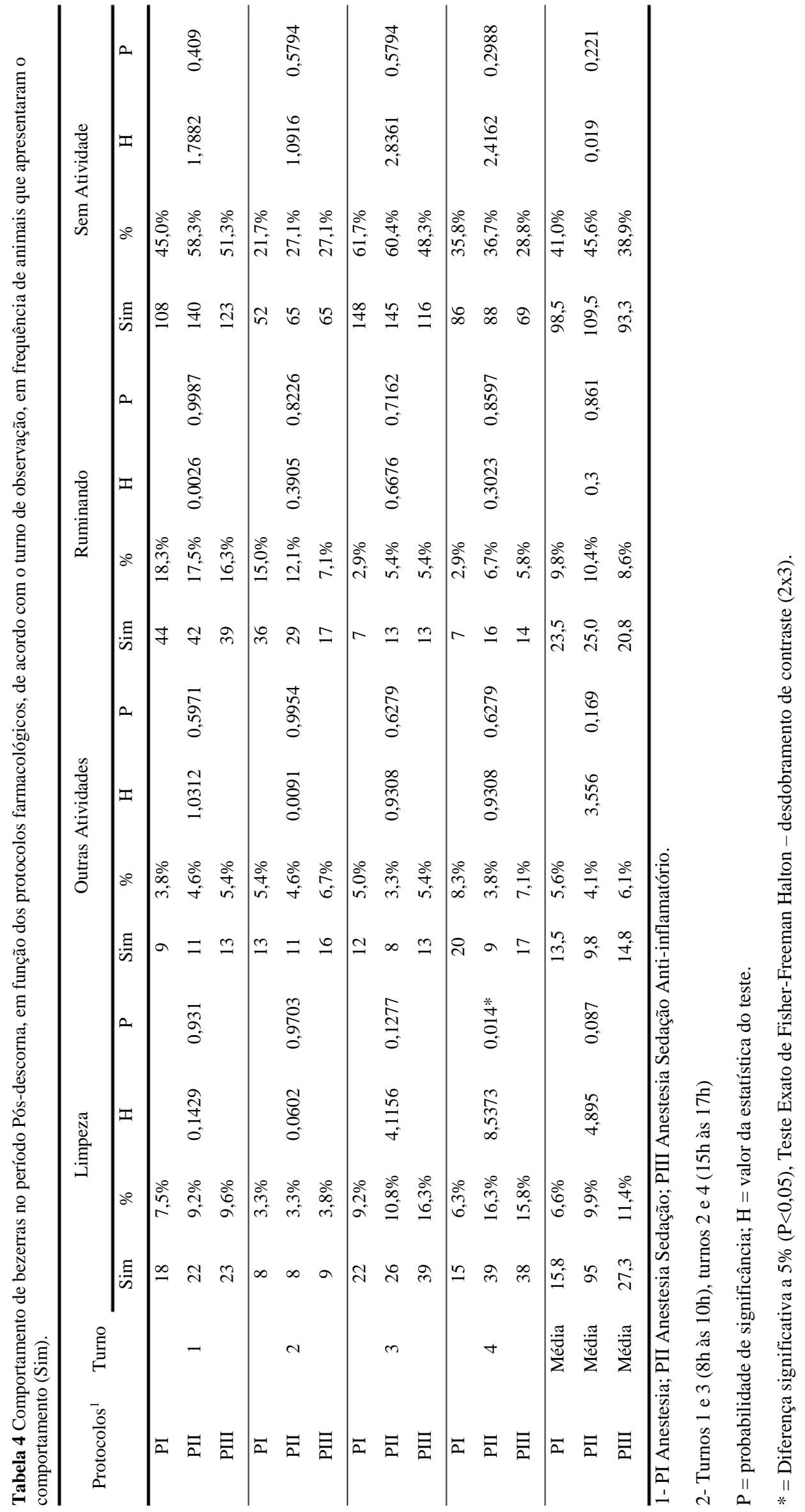


três protocolos estudados. No entanto, segundo a AVMA (2014) o nervo cornual representa um ramo do nervo trigêmeo e proporciona a sensibilidade da região do corno. Sendo assim, a injeção de anestésico local em torno deste nervo dessensibiliza esta área, sendo um procedimento anestésico importante no controle da dor na descorna de bezerros realizada com ou sem ferro quente.

A realização da descorna em bovinos é regulamentada em alguns países, como EUA, Canadá, Reino Unido, Austrália e Nova Zelândia. No Manual de Legislação do sistema CFMV/CRMVs, na Resolução no 877/2008 do Conselho Federal de Medicina Veterinária foi normatizado os procedimentos cirúrgicos nos animais de produção, incluindo a descorna. Embora esta resolução esteja bem definida, faz-se necessária a realização estudos para promover o refinamento dos procedimentos de descorna através do desenvolvimento de protocolos farmacológicos e não farmacológicos eficientes no controle da dor durante e após este procedimento. Outro fator importante que deve ser levado em consideração diz respeito ao tratamento dos animais no pós-operatório, sendo às vezes, necessária aplicação de antibióticos, antiinflamatórios sedativos e analgésicos, além do tratamento da ferida operatória até completar a evolução cicatricial, evitando complicações secundárias como infecções, sinusites e miíases infiltrativas que comprometem o bem-estar animal. No presente trabalho todo o período pósoperatório foi acompanhado diariamente, durante horários pré-determinados, até completar toda evolução cicatricial, o que propiciou boas condições clínicas aos animais.

Alguns estudos demonstram que durante a descorna foi evidenciado alterações comportamentais caracterizadas por esquivar com os membros, movimentação da cabeça e abanar a cauda, enquanto que no período pós-descorna os indicadores de dor foram caracterizados por alterações comportamentais como esfregar a cabeça sobre obstáculos, balançar a cabeça, extensão do pescoço, abanar das orelhas e cauda e alterações da ruminação (Faulkner \& Weary, 2000, Stilwell et al., 2007, Sutherland et al., $\underline{2002 b}$ ). No presente trabalho a avaliação do comportamento dos animais no período pósdescorna mostrou diferença significativa dos animais submetidos aos protocolos II ou III apresentando menor número de animais deitados quando comparado ao protocolo I, ao longo dos períodos. Ainda, no protocolo II houve diferença significativa quanto ao aumento de ingestão de alimento e autolimpeza corporal, nos períodos avaliados. Isto evidencia maior atividade dos animais e, consequentemente, melhoria no bemestar quando submetidos aos protocolos II e III, sugerindo uma condição clínica melhor. A associação do anti-inflamatório flunixinameglumina com o sedativo cloridrato de xilazina e anestesia local favoreceu o controle da dor dos animais deste grupo, sugerindo alívio por período mais prolongado. No protocolo III aplicou-se o anti-inflamatório flunixina-meglumina o qual quando associado ao sedativo cloridrato de xilazina e ao anestésico local cloridrato de lidocaína potencializou e prolongou o controle da dor contribuindo, assim, na melhoria da condição clínica dos animais deste grupo, sugerindo alívio da dor por um período mais longo. A presença de dor ou estresse nos animais pode ser evidenciada por manifestações comportamentais anormais ou por mudança na incidência do comportamento normal (Sylvester et al., 2004). A aplicação isolada de anestesia local não é efetiva para controlar a dor na descorna (Stewart et al., 2009). Ao estudar o comportamento de bezerros submetidos a descorna com ou sem aplicação de anestesia local observou-se que a realização da descorna sem aplicação prévia de anestesia local é um procedimento doloroso, notadamente nas primeiras seis horas, destacando a importância do controle da dor durante este período (Stewart et al., 2009).

O método de descorna com ferro quente é um dos mais utilizados na prática de campo. Ao estudar as respostas comportamentais e fisiológicas de bezerros descornados Vickers et al. (2005) evidenciaram sofrimento dos animais durante a contenção física, necessária para realizar a descorna, bem como a dor durante e após a realização da mesma, demonstraram ainda que a dor poderia ser controlada utilizando o bloqueio do nervo cornual com uso de anestesia local. No procedimento de descorna com ferro quente em bezerros de quatro a seis meses de idade verificaram alterações comportamentais como aumento dos movimentos da cauda e da cabeça, debatendo-se com maior frequência e elevação da concentração plasmática de vasopressina, ACTH e cortisol, verificaram ainda que estas alterações foram evitadas quando foi realizada anestesia local (Graf \& Senn, 1999). A combinação de anestesia local com anti-inflamatório é mais efetiva no controle da dor aguda, durante e após 
descorna de bezerros (Stafford \& Mellor, 2005). $\mathrm{Na}$ descorna com ferro quente o alívio da dor pósoperatória pode ser mais prolongado através do uso de anti-inflamatórios (Milligan et al., 2004).

Durante o procedimento de descorna do presente trabalho verificou-se nos protocolos estudados altas frequências nos comportamentos variando de $70 \%$ a $90 \%$ na cabeça, de 20 a $50 \%$ no temperamento e 20 a $70 \%$ nas alterações na cauda, demonstrando o comprometimento do bem-estar dos animais. Apesar destes resultados novos estudos devem ser conduzidos para avaliar de forma mais detalhada a etologia clínica dos animais, incluindo outros indicadores de dor. Além dos indicadores comportamentais existem os indicadores fisiológicos que são utilizados também para avaliar a dor na descorna de bezerros. No estudo realizado por Sylvester et al. (2004) foi verificado diminuição da ruminação durante as primeiras seis horas após a descorna. Esta alteração fisiológica foi eliminada no período em que os animais estavam submetidos à anestesia local, assim, este indicador confirma uma relação inversa entre ruminação e dor, sugerindo ser um indicador sensível, demonstrou ainda que a descorna é uma experiência dolorosa e que a dor dura aproximadamente seis horas, entretanto, quando submetidos à ação da anestesia local a dor é controlada durante duas horas.

A técnica da termocauterização associada à aplicação de anestésico local na descorna de bezerros foi estudada por outros autores. No trabalho realizado por Sutherland et al. (2002a) verificou-se que esta técnica controlou a hemorragia em todos os animais e aumentou o período de alívio da dor, até o término da ação do anestésico local. Demonstraram também que apesar da presença de necrose ocorrida não houve complicações de feridas operatórias em nenhum animal. Enfatizaram também que durante a termocauterização da ferida a contenção da cabeça dos animais, auxiliada com as mãos do operador, não apresentava segurança, não sendo recomendada, e que o ideal seria conter os animais na guilhotina, semelhante às do tronco de contenção. No entanto, no presente trabalho, foi observado que a contenção manual da cabeça durante o procedimento de descorna é imprescindível para realizar os procedimentos com segurança e evitar acidentes com os animais e com os operadores.

A utilização do anti-inflamatório não esteroide cetoprofeno associado à anestesia local na descorna com ferro quente em bezerros leiteiros observou-se que o nível de cortisol sérico foi significativamente mais baixo nos animais tratados com anti-inflamatório associado à aplicação de anestésico local (Milligan et al., 2004). Assim, demonstrando que a administração de um anti-inflamatório associado a um anestésico local atenua a dor inflamatória, em decorrência da lesão tecidual e da resposta inflamatória que os animais sentem no período pós-descorna. No estudo realizado por Stewart et al. (2009) foi verificado que o controle da dor de bezerros descornados com ferro quente foi mais efetivo quando usou aplicação de anestésico local combinado com o anti-inflamatório, destacaram a necessidade de melhorar o entendimento do mecanismo de respostas da inflamação e como estas podem ser usadas para compreender os estudos da dor, medo e estresse em animais de produção. No trabalho conduzido por Faulkner \& Weary (2000) foi demonstrado que a administração do anti-inflamatório no leite ministrado aos bezerros durante a amamentação no período pós-descorna reduziu as evidências de indicadores comportamentais de dor evidenciaram também a necessidade da combinação do antiinflamatório com anestesia local e sedativa para o controle efetivo da dor nos animais submetidos a este procedimento.

Ao comparar a descorna de bezerras utilizando guilhotina, ferro quente e pasta cáustica no comportamento e níveis plasmáticos de cortisol sérico verificou-se que a descorna com ferro quente provocou dor intensa e que os animais se debateram mais que aqueles descornados com uso da guilhotina ou pasta cáustica (Stilwell et al., 2007). Entretanto, não houve diferenças comportamentais entre os métodos estudados, tampouco nos níveis de cortisol antes e após descorna, de forma que tanto a descorna com uso de guilhotina e ferro quente quanto o uso da pasta cáustica causa dor intensa. No entanto, os autores não utilizaram nenhum procedimento farmacológico para controlar a dor e processo inflamatório. O comportamento de bezerras descornadas com uso de pasta cáustica na ação do analgésico cloridrato de tramadol demonstrou-se que os indicadores comportamentais são adequados para avaliar a intensidade de dor e a descorna com pasta com a pasta cáustica causa dor intensa e que o cloridrato de tramadol não foi eficaz no controle da dor (Braz, 2010).

No Canadá foi realizado um estudo com objetivo de comparar as práticas de descorna entre 
veterinários e produtores de leite. Neste estudo foi demonstrado melhorias nas práticas de controle da dor e ambos perceberam que o procedimento era doloroso e que necessitava de melhorias (Winder et al., 2016). Estudos similares foram realizados na Finlândia por Hokkanen et al. (2011), sendo que neste trabalho os autores enfatizaram ainda a necessidade de controlar a dor no período prédescorna. No Brasil o controle da dor em bezerros submetidos aos procedimentos de descorna ainda continua sendo um desafio. A literatura científica vem evoluindo nesta área e vem mostrando resultados promissores. Entretanto, na prática de campo percebe-se a necessidade de aprimoramento dos protocolos farmacológicos e melhoria na metodologia de manejo de contenção durante e após este procedimento.

\section{Conclusões}

Nas condições em que o estudo foi realizado conclui-se que os animais submetidos aos protocolos II e III evidenciaram maior atividade de ingestão de água e alimentos, ruminação e autolimpeza corporal, indicando que estes protocolos foram mais eficientes no controle da dor no período pós-operatório contribuindo assim no bem-estar dos animais. A metodologia utilizada na contenção dos bezerros em posição quadrupedal durante os procedimentos de descorna foi adequada para avaliar o comportamento e evitar acidentes por queimadura com ferro quente nos animais e nos operadores, podendo ser utilizada em outros estudos. Apesar da importância destes resultados faz-se necessário a realização de novos estudos, visando aprimorar a avaliação da dor no momento da descorna e intensificar o refinamento deste procedimento.

\section{Agradecimentos}

Ao Pesquisador Cientifico Eder Pinatti, aos Funcionários de Apoio a Pesquisa Científica e Tecnológica do Instituto de Zootecnia SAA/SP Srs. Hiram R. Chaves, Rita de Cássia B. Tognin, Gilmar de A. Gaia e Maria Vandenira de L. Souza; ao bolsista Arno Willian Cren Filho e a mestranda em Produção Animal Sustentável Ana Cláudia Koki Sampaio.

\section{Referências bibliográficas}

Abrahamsen, E. J. 2008. Ruminant field anesthesia. Veterinary Clinics: Food Animal Practice, 24, 429-441.
AVMA. 2014. - Welfare implications of the dehorning and disbudding of cattle. American Veterinary Medical Association., Washington, USA.

Braz, M. R. 2010. O efeito do tramadol na dor da descorna com pasta cáustica em vitelos. Faculdade de Medicina Veterinária. Universidade Técnica de Lisboa, Lisboa, Portugal.

Caray, D., Roches, A. B., Frouja, S., Andanson, S. \& Veissier, I. 2015. Hot-iron disbudding: stress responses and behavior of 1-and 4-week-old calves receiving anti-inflammatory analgesia without or with sedation using xylazine. Livestock Science, 179, 22-28.

Faulkner, P. M. \& Weary, D. M. 2000. Reducing pain after dehorning in dairy calves. Journal of dairy science, 83, 2037-2041.

Graf, B. \& Senn, M. 1999. Behavioural and physiological responses of calves to dehorning by heat cauterization with or without local anaesthesia. Applied Animal Behaviour Science, 62, 153-171.

Hokkanen, A.-H., Korhonen, T., Pastell, M., Valros, A., Vainio, O. \& Hänninen, L. 2011. Perceptions and practices of Finnish dairy producers regarding disbudding of calves. Journal of Dairy Science, 98, 823-631.

Lorena, S. E. R. S. 2010. Estudo demográfico sobre as condutas de avaliação e tratamento da dor dos médicos veterinários brasileiros no período perioperatório de grandes e pequenos animais. Faculdade de Medicina Veterinária e Zootecnia. Universidade Estadual Paulista Júlio de Mesquita Filho, Botucatu, São Paulo.

Luna, S. P. L. 2006. Dor e sofrimento animal. In: Rivera, E. A. B., Amaral, M. H. \& Nascimento, V. D. (eds.) Ética e Bioética Aplicada à Medicina Veterinária. Goiás. .

Martin, P., Bateson, P. P. G. \& Bateson, P. 1993. Measuring behaviour: an introductory guide. Cambridge University Press, Cambridge, , UK.

Massone, F. 1999. Anestesiologia veterinária. Guanabara Koogan, Rio de Janeiro.

Milligan, B. N., Duffield, T. \& Lissemore, K. 2004. The utility of ketoprofen for alleviating pain following dehorning in young dairy calves. The Canadian Veterinary Journal, 45, 140-143.

Misch, L. J., Duffield, T. F., Millman, S. T. \& Lissemore, K. D. 2007. An investigation into the practices of dairy producers and 
veterinarians in dehorning dairy calves in Ontario. The Canadian Veterinary Journal, 48, 1249-1254.

Moggy, M. A., Pajor, E. A., Thurston, W. E., Parker, S., Greter, A. M., SchwartzkopfGenswein, K. S., Campbell, J. R. \& Windeyer, M. C. 2017. Management practices associated with pain in cattle on western Canadian cowcalf operations: A mixed methods study. Journal of Animal Science, 95, 958-969.

SAS. 2004. SAS/STAT User guide, Version 9.1.2. SAS Institute Inc, Cary, NC, USA.

Stafford, K. J. \& Mellor, D. J. 2005. Dehorning and disbudding distress and its alleviation in calves. The Veterinary Journal, 169, 337-349.

Stewart, M., Stookey, J. M., Stafford, K. J., Tucker, C. B., Rogers, A. R., Dowling, S. K., Verkerk, G. A., Schaefer, A. L. \& Webster, J. R. 2009. Effects of local anesthetic and a nonsteroidal antiinflammatory drug on pain responses of dairy calves to hot-iron dehorning. Journal of Dairy Science, 92, 15121519.

Stilwell, G., Lima, M. S. \& Broom, D. M. 2007. Comparing the effect of three different disbudding methods on behaviour and plasma cortisol of calves. Revista Portuguesa de Ciências Veterinárias, 102, 281-288.

Sutherland, M. A., Mellor, D. J., Stafford, K. J., Gregory, N. G., Bruce, R. A. \& Ward, R. N. 2002a. Cortisol responses to dehorning of calves given a 5-h local anaesthetic regimen plus phenylbutazone, ketoprofen, or adrenocorticotropic hormone prior to dehorning. Research in Veterinary Science, 73, 115-123.

Sutherland, M. A., Mellor, D. J., Stafford, K. J., Gregory, N. G., Bruce, R. A. \& Ward, R. N. 2002b. Effect of local anaesthetic combined with wound cauterisation on the cortisol response to dehorning in calves. Australian Veterinary Journal, 80, 165-167.

Sylvester, S. P., Stafford, K. J., Mellor, D. J., Bruce, R. A. \& Ward, R. N. 2004. Behavioural responses of calves to amputation dehorning with and without local anaesthesia. Australian Veterinary Journal, 82, 697-700.

Vickers, K. J., Niel, L., Kiehlbauch, L. M. \& Weary, D. M. 2005. Calf response to caustic paste and hot-iron dehorning using sedation with and without local anesthetic. Journal of Dairy Science, 88, 1454-1459.

Winder, C. B., LeBlanc, S. J., Haley, D. B., Lissemore, K. D., Godkin, M. A. \& Duffield, T. F. 2016. Practices for the disbudding and dehorning of dairy calves by veterinarians and dairy producers in Ontario, Canada. Journal of Dairy Science, 99, 10161-10173.

Article History:

Received 21 February 2018

Accepted 16 March 2018

Available online 10 April 2018

License information: This is an open-access article distributed under the terms of the Creative Commons Attribution License 4.0, which permits unrestricted use, distribution, and reproduction in any medium, provided the original work is properly cited. 